\title{
RNF180 mediates STAT3 activity by regulating the expression of RhoC via the proteasomal pathway in gastric cancer cells
}

\section{Zizhen Wu}

tianjin tumor hospital

Huifang Liu

Tianjin Tumor Hospital

Weilin Sun

Tianjin Tumor Hospital

Yingxin Du

Tianjin Tumor Hospital

Wenting $\mathrm{He}$

tianjin tumor hospital

Shiwei Guo

Tianjin Tumor Hospital

Liqiao Chen

Tianjin Tumor Hospital

Zhenzhen Zhao

Tianjin Tumor Hospital

Pengliang Wang

Tianjin Tumor Hospital

Han Liang

Tianjin Medical University

Jingyu Deng ( $\nabla$ dengery@126.com )

Tianjin Tumor Hospital

\section{Research}

Keywords: stomach, neoplasm, progression, RNF180, RhoC, STAT3

Posted Date: April 8th, 2020

DOI: https://doi.org/10.21203/rs.3.rs-21786/v1 
License: (c) (i) This work is licensed under a Creative Commons Attribution 4.0 International License. Read Full License 


\section{Abstract}

\section{Background}

Ring finger protein 180 (RNF180) is an important member of the E3 ubiquitin ligase family. As a tumor suppressor gene, RNF180 is significantly associated with the prognosis of patients with gastric cancer (GC) and can inhibit the proliferation, invasion, and migration of GC cells. Signal transducer and activator of transcription 3 (STAT3) is considered one of the most common oncogenes in human cancers with a key role in GC progression. In this study, we explored the relationship between RNF180 and STAT3 in GC cells.

Methods

The RNF180 gene was significantly overexpressed to observe its regulatory activity on STAT3 and pSTAT3 in GC cells. We then explored the molecular signaling pathways by which RNF180 could potentially regulate STAT3 through transcriptomics and proteomics experiments.

Results

RNF180 overexpression could suppress STAT3 phosphorylation in GC cells. Ubiquitin label-free experiments showed that the ubiquitination level of Ras homolog gene family member $C$ (RhoC) is significantly increased in GC cells transfected with an RNF180 expression vector (RNF180-GFP vector) compared with cells transfected with an empty vector (vehicle vector). We subsequently demonstrated that RNF180 could directly combine with RhoC and promote the ubiquitination and degradation of RhoC protein in GC cells. The phosphorylation level of STAT3 significantly decreased in GC cells after RhoC knockdown using small hairpin RNA(shRNA).

\section{Conclusions}

RNF180 could inhibit GC progression by reducing the phosphorylation of STAT3 via the ubiquitination and degradation of RhoC protein in GC cells. Thus, the protein may be considered a novel therapeutic target for patients with GC.

\section{Background}

Gastric cancer (GC) is one of the most common malignant tumor and the third leading cause of cancerrelated mortality worldwide ${ }^{1}$. Diagnostic and therapeutic strategies for GC have seen remarkable improvements over the years, however, the 5-year survival rate of patients with the disease remains low due to the high incidence metastasis. While radical surgery is considered the most effective treatment for GC, targeted therapy is gradually showing excellent therapeutic effects, especially for patients with unresectable GC. Therefore, the molecular mechanisms involved in the development and progression of GC should be explored. 
Ring finger protein 180 (RNF180) is a novel discovered member of the ring finger protein family and has been confirmed to be ubiquitin ligase (E3), which is involved in many important physiological processes in vivo including cell growth, differentiation, and tumorigenesis. As a tumor suppressor gene, RNF180 also has a certain expression level in the cells of normal body, and its expression also have important physiological functions. Under abnormal conditions, the body is affected by a variety of physical, chemical and biological factors, resulting in a decline in its expression level or the corresponding protein function abnormal, will make its normal physiological function decline or loss, which will lead to the occurrence of a series of diseases, including the occurrence of malignant tumors ${ }^{2}$. In our previous study, we demonstrated that RNF180 protein expression in tumor tissues is significantly associated with the overall survival (OS) of 134 patients with GC after curative gastrectomy ${ }^{3}$. Studies have shown that high levels of vascular endothelial growth factor-C and D (VEGF-C and D) expression are closely related to the density of lymphatic vessels and lymph node metastasis in GC and significantly associated with the prognosis of these patients 4,5 . In addition, we found an obvious negative correlation between RNF180 protein expression in tumor tissues and lymph node metastasis stage in patients with GC ${ }^{3}$.

Signal transducer and activator of transcription 3 (STAT3), an important oncogene is widely reported in many malignant tumors and its Activation involved in the development and progression of a variety of cancers, including $\mathrm{GC}^{6-8}$. We previously demonstrated that the expression level of STAT3 in the GC tissues was significantly correlated with lymph node metastasis rate ${ }^{9,10}$. Moreover, we found that STAT3 knockdown could significantly reduce the protein expression of VEGF-D and reduce the expression of VEGF-C to some extent ${ }^{11}$.RNF180 and STAT3 play considerable roles in GC, but their specific mechanisms remain unclear. Considering that RNF180 and STAT3 are associated with lymph node metastasis in GC and could regulate the expression of VEGF-C/D, we suppose a certain relationship between these proteins. In this study, we utilized human tissues, ubiquitination label-free quantitative proteomic analysis, and in vivo and in vitro assays to investigate the possible role of RNF180 in regulating STAT3 in GC. We also conducted ubiquitination label-free quantitative proteomic analysis to identify the oncogene RhoC. The abnormally high expression of RhoC has been associated with the development of malignant tumors, such as head and neck cancers, ovarian cancer, and GC, and could promote the proliferation, invasion, and migration of cancer cells ${ }^{12-14}$. The results demonstrated that RNF180 plays a critical role in mediating the activity of STAT3 by regulating RhoC expression via the proteasomal pathway in GC cells.

\section{Materials And Methods}

\section{Tissue Sample Collection and Follow-up}

We collected tumor and adjacent nontumor tissues from 113 patients with GC who underwent curative gastrectomy between January 2004 and September 2007 at Tianjin Medical University Cancer Hospital (Tianjin, China), Xijing Hospital of Air Force Medical University (Xi'an, China), and Renji Hospital of Shanghai Jiao Tong University School of Medicine (Shanghai, China). After curative surgery, all patients 
received standard followed up according to the guideline. The median follow-up for the entire cohort was 36 months (range: 2-77 months). The follow-up of all patients included in this study was completed in December 2010. Oral and written informed consent to use their tissues and clinicopathological information were provided by all patients.

\section{Cell lines}

Gastric cancer cell lines HGC-27 and SGC-7901 were purchased from the Type Culture Collection of the Chinese Academy of Sciences, (Shanghai, China). Cells were cultured in RPMI1640 medium (Thermo Electron Corporation, Beijing, China) supplemented with $10 \%$ fetal bovine serum (FBS) and maintained at $37^{\circ} \mathrm{C}$ in a 5\% CO2 atmosphere. HEK293T cell line was obtained from American Type Culture Collection (ATCC) and at $37 \mathrm{C}$ in 5\% CO2 in high glucose Dulbecco's Modified Eagle's medium(DMEM) supplemented with $10 \%$ fetal bovine serum.

\section{Immunohistochemistry}

The expression of RNF180 and RhoC was observed in 113 pairs of tumor and adjacent nontumor tissues by Immunohistochemistry (IHC). Mouse anti-RNF180 antibody (1:150) (H00285671-M05; Abnova,) and mouse anti-RhoC (1:50) (sc-393090; Santa Cruz Biotechnology) were used as primary antibodies. Positive cell rates and coloring intensity were scored separately. Then, the IHC staining score was obtained by using the H-score system. The cytoplasmic expression of RNF180 was assessed by assigning scores to the average intensity of positive tumor cells ${ }^{15}$.

Quantitative Reverse Transcription-Polymerase Chain Reaction (qRT-PCR).Total RNA was isolated from cells by using TRIzol reagent (\#15596018, Thermo Fisher). Primescript ${ }^{\text {TM }}$ RT Master Mix (RR036A; Takara) was used to synthesize the complementary DNA. $2 \mu \mathrm{g}$ of RNA was added to the real time PCR, with the fifinal primer concentration being $0.5 \mu \mathrm{M}$. The PCR was performed under the following condition: reverse transcription at $42 \circ \mathrm{C}$ for $60 \mathrm{~min}$, amplifification for 30 cycles at $94 \circ \mathrm{C}$ for $30 \mathrm{~s}, 58 \circ \mathrm{C}$ for $50 \mathrm{~s}$, and $72 \circ \mathrm{C}$ for $50 \mathrm{~s}$. The primer sequences used are given in Supplementary Table 1.

\section{Western Blot}

Western blot was performed following standard methods. The primary antibodies used for Western blot are as follows: mouse anti-RNF180 antibody (1:1000) (H00285671-M05; Abnova), mouse anti-RhoC (1:50) (sc-393090; Santa Cruz Biotechnology), rabbit anti-pSTAT3 antibody (1:2000) (\#9145; Cell Signaling Technology), rabbit anti-STAT3 antibody (1:1000) (\#12640; Cell Signaling Technology), rabbit anti-flag antibody (1:1000) (\#2272S; Cell Signaling Technology), rabbit anti-HA antibody (1:1000) (\#3724T; Cell Signaling Technology), rabbit anti-myc antibody (1:1000) (\#14793S; Cell Signaling Technology), and rabbit anti- $\beta$-actin antibody (1:1000) (GTX109639; GeneTex).

\section{Vector Construction and Transfection}

The expression level of RNF180 was upregulated by using plasmid (pCMV6-AC-GFP-RNF180), and RhoC was knocked down by using short hairpin RNA (shRNA). An empty vector was transfected into the cells at 
the same time. Lipofectamine ${ }^{\text {TM }} 3000$ was used to transfect the shRNAs into the GC cells. Transfection efficiency was measured by using Western blot.

\section{Cell Viability Assay}

Cell viability was assessed by performing cell viability assays with a cell counting kit (CCK8). Cellls were plated in 96-well culture plate with $1 \times 10^{3}$ cells per well, and $10 \mu \mathrm{L}$ of CCK8 reagent was added to each well once a day. The optical density of the solution at $450 \mathrm{~nm}$ was measured after $3 \mathrm{~h}$.

\section{Colony Formation Assay}

Colony formation assay was used to detect the proliferation ability of GC cells. We seeded 1000 cells into each well of a 6-well plate and incubated at $37 \circ \mathrm{C}$ for 12-14 days. The medium was changed at regular intervals until a macroscopic clone formed.

\section{Wound Healing Assay and Transwell Tumor Cell Invasion Assay}

Wound healing assay was performed on transfected GC cells as described in our previous study ${ }^{16}$. Matrigel $^{\mathrm{TM}}$ (Sigma) invasion experiments were performed on transfected GC cells as described by Jingyu et al ${ }^{16}$

\section{Animal Experiment}

To explore the effect of RhoC on GC cell proliferation in vivo, we purchased seven female 4-week-old BALB/c mice (SPF [Beijing] Biotechnology Co., Ltd.). All mice were bred in the animal facility of Tianjin Medical University Cancer Hospital. A total of $10^{6}$ HGC-7901-shControl and HGC-7901-shRhoC cells were subcutaneously xenografted into the mice. The length $(I)$ and width $(\mathrm{w})$ of the tumors were measured after 3 weeks, and the tumor volume was calculated as $v=1 / 2 \times 1 \times w^{2}$.

\section{Ubiquitination Label-free Quantitative Proteomic Analysis}

HGC-27 cells were transfected with the RNF180 expression and empty vectors. Total proteins from each sample were extracted via SDT lysis and separated with 12.5\% SDS-PAGE gel. Protein bands were visualized by Coomassie Blue R-250 staining. Peptides were collected with trypsin, desalted, concentrated, and reconstituted. The peptide content was estimated by determining the UV spectral density at $280 \mathrm{~nm}$. Liquid chromatography-tandem mass spectrometry was performed at Shanghai Applied Protein Technology (Shanghai, China) using a Q Exactive mass spectrometer (Thermo Scientific).

\section{Co-immunoprecipitation Assay}

Total proteins were extracted $72 \mathrm{~h}$ after HEK293T cells were co-transfected with the indicated plasmids. The protein lysates were incubated with anti-FLAG or anti-MYC agarose beads at $4{ }^{\circ} \mathrm{C}$ overnight, centrifuged at $3000 \times g$ for $5 \mathrm{~min}$, and then washed with lysis buffer thrice. Finally, the precipitates were collected for subsequent Western blot analysis. The plasmid of HA-ubiquitin was co-transfected into 
HEK293T cells according to the above experimental methods, and precipitates were collected for subsequent Western blot analysis.

\section{Protein Stability and Degradation Experiment}

Cycloheximide (CHX), a protein synthesis inhibitor, could act on the large subunit of eukaryotic ribosome, inhibit transpeptidase, block peptide chain extension, and further inhibit protein synthesis. Approximately $30 \mathrm{~h}$ after transfection, $\mathrm{CHX}$ (HY12320; MedChemExpress) was added to the GC cell culture medium at a concentration of $100 \mu \mathrm{g} / \mathrm{ml}$, and the total protein was extracted for Western blot analysis.MG132, a proteasome inhibitor, could inhibit protein degradation in a proteasome-dependent manner. Approximately $48 \mathrm{~h}$ after transfection, $5 \mu \mathrm{M}$ MG132 (HY12359; MedChemExpress) was added to the GC cell culture medium, and the same amount of dimethyl sulfoxide (DMSO) was added to the control group. Then, $11 \mathrm{~h}$ after this treatment, the total protein was extracted for Western blot analysis.

\section{Statistical Analysis}

We analyzed the results by using t-test to compare statistical differences between two groups or one-way analysis of variance to compare differences among three groups. Statistical analyses were performed by using IBM SPSS Statistics (version 24.0; Armonk, NY, USA) and GraphPad Prism Version 8.0 software. Overall survival (OS) was using Kaplan-Meier method and log-rank test performed to determine significance. The multivariate analysis of OS was performed by the Cox proportional hazard model with forward step procedures. A two tailed $P$ value $<0.05$ was considered statistically significant.

\section{Results}

\section{Patient Outcomes Related to RNF180 Expression}

We measured the expression of RNF180 in 113 pairs of tumor and adjacent non-tumor tissues by IHC (Figs. 1A-B). The results of univariate and multivariate survival analyses of 113 patients with GC are summarized in Table 1. The median OS of all patients with GC was 36 months, and 25 patients were alive at the end of follow-up. Kaplan-Meier analysis showed that patients with negative (no/weak) RNF180 protein expression had poorer survival than those with positive (moderate/high) RNF180 protein expression (Fig. 1C). RNF180 protein expression in GC tissues was identified as an independent predictor of outcome (hazard ratio $[H R] 0.628 ; P=0.006), p N$ stage (HR 1.253; $P=0.005)$ and tumor location (HR $1.218 ; P=0.033)$. RNF180 negativity indicated a tendency of poor prognosis in patients with $G$.

\section{Up-regulation of RNF180 Increased the Ubiquitination Level of RhoC}

To determine substrates that may be regulated by RNF180, we measured the ubiquitination level of proteins extracted from HGC-27-vector and HGC-27-RNF180 cells. Ubiquitination label-free quantitative proteomic analysis revealed that the ubiquitination level of 12 proteins is significantly increased 
compared with the control group by upregulating RNF180 (fold change $\geq 1.5, P<0.05$ ) (Fig. 1D). Among these 12 proteins, we chose RhoC for further analysis because previous reports indicated that expression of RhoC is associated with metastasis of Gastric cancer ${ }^{17}$.

\section{RNF180 and RhoC Expression Patterns in Human Tissue Microarrays}

We measured the protein expression levels of RNF180 and RhoC in 113 pairs of tumor and adjacent nontumor tissues by IHC (Figs. 1E). RNF180 protein expression levels in GC were significantly lower than those in normal gastric tissues $(P=0.0002)$. By contrast, $G C$ tissues showed higher expression levels of RhoC compared with normal gastric tissues $(P=0.0011)$. The IHC analysis results of RNF180 and RhoC are summarized in Figs. 1F, respectively.

\section{RNF180 Promotes RhoC Degradation}

RNF180 has been demonstrated to be a ubiquitin ligase for several substrates ${ }^{32}$. To support our hypothesis that RNF180 could also function as an ubiquitinating ligase to promote the RhoC degradation, we transfected increasing concentrations of RNF180 into HEK293T cells. The protein level of RhoC was gradually reduced by RNF180 in a dose-dependent manner (Fig. 2A). However, RhoC mRNA levels did not show significant differences (Fig. 2B), thereby indicating that RNF180 regulates RhoC expression at the posttranslational level. CHX pulse-chase assay was performed to examine the function of RNF180 in RhoC.We observed that RNF180 overexpression could remarkably decrease RhoC protein levels and reduce its half-life (Fig. 2C). To confirm whether RhoC is a direct substrate of RNF180, we performed coimmunoprecipitation assay. As shown in Fig. 2D, RhoC is a direct substrate of RNF180. A reciprocal coimmunoprecipitation experiment was then carried out to further define their interaction (Fig. 2E), and an in vivo ubiquitination assay was performed to confirm whether the ubiquitination level of RhoC is greatly increased by RNF180 (Fig. 2F). Taken together, our findings indicate that RNF180 is an ubiquitin ligase targeting RhoC for ubiquitination and degradation.

\section{RNF180 Regulates RhoC Stability in GC}

We explored the influence of RNF180 on the degradation of RhoC protein by utilizing CHX, which could inhibit protein synthesis. The results showed that the protein band gradually weakens with the passage of time after $\mathrm{CHX}$ treatment and that this phenomenon is more pronounced in the RNF180 group than in the vector group in HGC-27 and SGC-7901 cells (Figs. 3A, 3B). Ubiquitination assay was performed with MG132 to explore the ubiquitination and degradation of RhoC by RNF180. As shown in Figs. 3C and 3D, the protein expression of RhoC in cells treated with MG132 was higher than that in cells treated with DMSO, thereby indicating that RNF180 could promote RhoC degradation through the ubiquitin proteasome system.

\section{RhoC Enhances the Proliferation, Migration, and Invasion of GC Cells and Tumor Growth in Mice}


To investigate the biological function of RhoC in GC cells, we used shRNA to transfect HGC-27 and SGC7901 cells and downregulate RhoC. The empty vector was also transfected as a control. As shown in Fig. 4A, B, protein expression levels were significantly decreased after plasmid transfection. The cell growth curve of CCK8 assay results showed that downregulated RhoC suppressed cell proliferation and viability.(Fig. 4C).Similarly,as shown in Fig. 4D, fewer colonies were formed in the downregulated RhoC group than in the control group.thus, cell viability was significantly weaker in the downregulated RhoC group than in the vector group. The size and weight of subcutaneous tumor transfected with shRNA RhoC was significantly decreased than that in tumor transfected with empty vector. RhoC appeared to remarkably enhance tumor growth in vivo (Fig. 4E).Wound healing and transwell assays were conducted to investigate the effect of RhoC on the migration and invasion ability of HGC-27 and SGC-7901 cells (Figs. 4F, 4G). Wound healing and transwell assays result showed that the migration distances of downregulated RhoC group were significantly reduced compared with that of the control group.Thus,the results revealed that the proliferation and migration ability of cells in the downregulated RhoC group was lower than that in the vector group.

\section{RNF180 Inhibits Constitutively Active STAT3 in GC}

Persistent activation of STAT3 contributes to uncontrolled cell proliferation, angiogenesis, apoptotic resistance, and prosurvival effects in cancer cells ${ }^{18}$. Western blot analysis of HGC-27 and SGC-7901 cells showed that the protein expression level of p-STAT3 (Y705) is obviously downregulated by RNF180 (Figs. 5A, B). In addition, knockdown of RhoC could reduce the activation level of STAT3 but not that of STAT3 (Figs. 4A, B). Inhibition of pSTAT3 resulting from RNF180 overexpression could be rescued by RhoC overexpression (Fig. 5E), likely because STAT3 is known as a RhoC downstream molecule with a critical role in regulating the interferon response pathway ${ }^{19}$. Based on the above results, we believe that RNF180 could inhibit STAT3 activation by degrading RhoC. We observed altered mRNA expression levels in genes downstream of the STAT3 signaling pathway in GC cells featuring STAT3 knockdown, including substantial decreases in MMP-2, MMP-14, VEGF-C, VEGF-D, and HGF levels compared with those in control GC cells (Supplementary Fig.). A schematic summarizing the effects of RNF180 on the inhibition of GC proliferation is shown in Fig. 6.

In summary, our findings suggest that RNF180 could reduce tumor metastasis in GC by inhibiting STAT3 activation via RhoC protein degradation through the ubiquitin proteasome system.

\section{Discussion}

In this study, we utilized human tissues, genome-wide transcriptome analysis, ubiquitination label-free quantitative proteomic analysis, and in vivo and in vitro assay to examine the role of RNF180 in suppressing STAT3 activity by degrading RhoC protein through the ubiquitin proteasome system.

The ubiquitin-proteasome system is one of the major systems involved in proteolysis in eukaryotic cells 20. E3 ubiquitin ligase is considered the most important component of this system because it can 
recognize substrates specifically ${ }^{21}$. Our own studies on RNF180 revealed that the newly discovered E3 ubiquitin ligase could inhibit the lymph node metastasis of $\mathrm{GC}^{3}$. In the present study, our findings demonstrated that RNF180 could bind with RhoC and increase its ubiquitination to promote protein degradation. We also found that the protein level of RhoC in GC cells decreases gradually after addition of $\mathrm{CHX}$ and that this phenomenon is more pronounced in the RNF180 upregulation group. Addition of the proteasome inhibitor MG132 inhibited the degradation of RhoC, which means RNF180 could modulate the proteolysis of RhoC through the ubiquitin-proteasome pathway.

As an important member of Rho family, RhoC is a guanosine triphosphatase that can modulate the proliferation, differentiation, and apoptosis of normal cells ${ }^{22-24}$. Previous studies have shown that the Rho-family of small GTPases plays an essential role in transmitting the VEGF signals downstream to angiogenesis ${ }^{25}$.Upon VEGF stimulation, c-Src is activated Src interacts with FAK and phosphorylated at Tyr-416; the Src/FAK complex further activates Rho GTPases ${ }^{26}$. The super-family of Rho-GTPases consists of various Rho specific insertion domains containing subfamilies such as Rho, Rac, Cdc-42, etc. RhoC expression and/or activity frequently is increased in various cancers ${ }^{27}$.Moreover, increased expression of RhoC may be involved in the metastasis of gastric cancer,and RhoC appears to be a good genetic marker of a metastatic potential ${ }^{28}$.It has also been reported that the abnormal high expression of RhoC was associated with the development of malignant tumors such as head and neck cancer, ovarian cancer and gastric cancer, it could promote the proliferation, invasion and migration of cancer cells ${ }^{29-}$ ${ }^{31}$.Islam M et al ${ }^{19}$ found that RhoC could regulate cancer stem cells (CSCs) by overexpressing the phosphorylation of STAT3. To further understand how RhoC regulates the expression of the GC cell transcription factors, we analyzed expression levels of the STAT3.Since the role of RhoC in STAT3 phosphorylation has not been established before in GC, we analyzed the phosphorylation of STAT3 tyr705 in the RhoC knockdown cell lines and the control and showed that there was a decrease in pSTAT3 tyr705 in the RhoC knockdown GC cell lines (Fig. 7C,D).Based on these results, we could conclude that RhoC promoted the proliferation and migration of GC cells through regulating the phosphorylation of STAT3. Apparently, knockdown of RhoC suppressed tumor proliferation and invasion(Fig. 6).Taken together, in this study, RNF180 is characterized as a tumor suppressor to inhibit tumor metastasis by reducing $\mathrm{RhoC}$ in gastric cancer.

Metastasis is closely associated with poor survival outcomes. As a well-known transcription factor, STAT3 is implicated in the proliferation, migration, and invasion of various tumor cells depending on its active form, PSTAT3 ${ }^{32}$. Activation of STAT3 transcription activity induces the expression of a wide range of target genes promoting key pro-oncogenic cellular functions, such as inflammation, proliferation, survival, invasion, and angiogenesis ${ }^{33}$. STAT3 is activated by phosphorylation of the Y705 position, followed by nuclear translocation of the phosphorylated proteins with subsequent activation of the transcription of specific downstream genes and metastasis of cancers ${ }^{34}$. Our results demonstrated decreased mRNA expression levels in GC cells featuring STAT3 knockdown and downregulation of several STAT3 target genes, such as MMP-2, MMP-14, VEGF-C, VEGF-D, and HGF. These genes play an important role in regulating cellular functions in cancer development and progression. 
Based on our findings, we constructed the signaling pathway by which RNF180 regulates the proliferation and invasion of gastric cancer (Fig. 8).RNF180 binds to the RhoC and degrade of RhoC protein by increasing its ubiquitination level, which can phosphorylate STAT3 and increase the protein level of $p$ STAT3(Y705).Phosphorylated STAT3 at tyr-705 is able to dimerize and then diffuse into the nucleus where it binds to the promoter region of nanog to switch on its expression.As a result, lowlevel of p-STAT3 could efficiently inhibit the proliferation and invasion of gastric cancer.In this way, an increased RNF180 expression in GC results in the inhibition of the core cell transcription factors that are needed for their proliferation and invasion. This in turn significantly decreases the tumors' ability to grow and metastasize to other body regions. The implications of these findings provide a fertile area of research in GC.Additional studies are needed to understand how RNF180 regulates the RhoC expression and what additional signaling molecules are involved.

\section{Conclusion}

In summary, our study reveals that RhoC is a substrate of RNF180 and that ubiquitination and degradation of RhoC by RNF180 could inhibit lymph node metastasis in GC.Thus, RNF180 may be a potential candidate for GC treatment,illustrating that RNF180 plays an important role in Gastric cancer and its propagation by modulating the phosphorylation state of STAT3. Considering the low expression of RNF180 in gastric cancer, efficiently inhibit RhoC expression might repress tumorigenesis and tumor metastasis and then improve the survival outcomes of patients, which still need further investigation. It seems to be a promising cure for metastatic tumor to develop small molecule inhibitors for RNF180 to target RNF180-decreased RhoC.With additional investigations and ongoing development of molecular therapy for gastric cancer, this may prove to be an important therapeutic target in the GC patient population.

\section{Abbreviations}

RNF180:Ring finger protein 180;GC:gastric cancer;STAT3:Signal transducer and activator of transcription 3;RhoC:Ras homolog gene family member C;shRNA:small hairpin RNA;OS:overall survival;VEGFC:vascular endothelial growth factor-C;VEGF-D:vascular endothelial growth factor-D;TMAs:tissue microarrays;IHC:Immunohistochemistry;CHX:Cycloheximide;DMSO:dimethyl sulfoxide;CSCs:cancer stem cells;MMP:matrix metalloproteinase;

\section{Declarations}

\section{Ethics approval and consent to participate}

This research was approved by the Ethics Committee of Tianjin Medical University Cancer Hospital, and Oral and written inform consents were obtained from all patients before enrolling in the research program. The in vivo assay using nude mice was approved by the Institutional Animal Care and Use Committee of Tianjin Medical University Cancer Hospital. 


\section{Consent for publication}

Not applicable.

\section{Availability of data and materials}

Not applicable.

\section{Competing interests}

The authors declare that they have no competing interests.

\section{Funding}

This study was supported in part by grants from the Program of National Natural Science Foundation of China (NO.81572372), National key R\&D Program of China (NO. 2016YFC1303202) and National key research and development program "precision medicine research" program (N0.2017YFC0908300).

\section{Authors' contributions}

*ZW, JD and HL are joint first authors; ZW and JD studied concept and design; ZW collected of data; ZW, HL, WS, YD, WH, SG, LC, ZZ and PW finished the experiments; ZW analyzed and interpreted of data; ZW and HL provided the technical and material support; ZW drafted of the manuscript; JD and HL critically revised of the manuscript for important intellectual content; $Z W$, JD performed the statistical analysis. All authors read and approved the final manuscript.

\section{Acknowledgements}

Not applicable.

\section{Authors' information}

Department of Gastroenterology, Tianjin Medical University Cancer Hospital, City Key Laboratory of Tianjin Cancer Center and National Clinical Research Center for Cancer, Tianjin, China;

\section{References}

1. Ferlay J, Soerjomataram I, Dikshit R, Eser S, Mathers C, Rebelo M, et al. Cancer incidence and mortality worldwide: Sources, methods and major patterns in GLOBOCAN 2012. INT J CANCER. 2015 Mar 1;136(5):E359-86.

2. Cheung KF, Lam CN, Wu K, Ng EK, Chong WW, Cheng AS et al. Characterization of the gene structure, functional significance, and clinical application of RNF180, a novel gene in gastric cancer. span>Cancer. 2012 Feb 15;118(4): $947-59$. 
3. Deng J, Liang H, Zhang R, Hou Y, Liu Y, Ying G, et al. Clinical and experimental role of ring finger protein 180 on lymph node metastasis and survival in gastric cancer. Br J Surg. 2016;03-01(4):407$16 . . . ; 103($.

4. Kigure W, Fujii T, Sutoh T, Morita H, Katoh T, Yajima RN, et al. The association of VEGF-C expression with tumor lymphatic vessel density and lymph node metastasis in patients with gastric cancer and gastrointestinal stromal tumor. Hepatogastroenterology. 2013-03-01;60(122):277-80.

5. Tanaka M, Kitadai Y, Kodama M, Shinagawa K, Sumida T, Tanaka S, et al. Potential role for vascular endothelial growth factor-D as an autocrine factor for human gastric carcinoma cells. CANCER SCI. 2010;10-01(10):2121-7... .;101(.

6. Jenkins BJ, Grail D, Nheu T, Najdovska M, Wang B, Waring P, et al. Hyperactivation of Stat3 in gp130 mutant mice promotes gastric hyperproliferation and desensitizes TGF-beta signaling. NAT MED. 2005-08-01;11(8):845-52.

7. Chen JF, Wu P, Xia R, Yang J, Huo XY, Gu DY, et al. STAT3-induced IncRNA HAGLROS overexpression contributes to the malignant progression of gastric cancer cells via mTOR signal-mediated inhibition of autophagy. MOL CANCER. 2018;01-12(1):6... . ; 17(.

8. Jiang Y, Zhou Z, Ji Y. Suppression of EGFR-STAT3 signaling inhibits tumorigenesis in a lung cancer cell line. INT J CLIN EXP MED. 2014-01-01;7(8):2096-9.

9. Deng JY, Sun D, Liu XY, Pan Y, Liang H. STAT-3 correlates with lymph node metastasis and cell survival in gastric cancer. World J Gastroenterol. 2010;11-14(42):5380-7.. . -;16(.

10. Deng J, Liang H, Zhang R, Sun D, Pan Y, Liu Y, et al. STAT3 is associated with lymph node metastasis in gastric cancer. Tumour Biol. 2013;10-01(5):2791-800.. . ; ;4(.

11. Deng J, Cui J, Jiang N, Zhang R, Zhang L, Hao X, et al. STAT3 regulation the expression of VEGF-D in HGC-27 gastric cancer cell. AM J TRANSL RES. 2014-01-20;6(6):756-67.

12. Zhao Y, Zheng HC, Chen S, Gou WF, Xiao LJ, Niu ZF. The role of RhoC in ovarian epithelial carcinoma: a marker for carcinogenesis, progression, prognosis, and target therapy. GYNECOL ONCOL. 2013;0901(3):570-8.. . - ;130(.

13. Jiang L, Wang WJ, Li ZW, Wang XZ. Downregulation of Piwil3 suppresses cell proliferation, migration and invasion in gastric cancer. CANCER BIOMARK. 2017;12-06(4):499-509.. . ;20(.

14. Datta J, Islam M, Dutta S, Roy S, Pan Q, Teknos TN. Suberoylanilide hydroxamic acid inhibits growth of head and neck cancer cell lines by reactivation of tumor suppressor microRNAs. ORAL ONCOL. 2016-05-01;56:32 - 9 .

15. Cheung K-F, Cleo NY, Lam K, Wu, Enders KO, Ng WWS, Chong ASL, Cheng, et al.Characterization of the Gene Structure,Functional Significance, and Clinical Application of RNF180, a Novel Gene in Gastric Cancer.Cancer. 2012 Feb 15;118(4):947 - 59.

16. Deng J, Guo J, Guo X, Hou Y, Xie X, Sun C,et al.Mediation of the malignant biological characteristics of gastric cancer cells by the methylated CpG islands in RNF180 DNA promoter.Oncotarget, $2016 \mathrm{Jul}$ 12;7(28):43461-43474. 
17. Kondo T, Sentani K, Oue N, Yoshida K, Nakayama H. Yasui W.Expression of RhoC Is Associated with Metastasis of Gastric. CarcinomasPathobiology. 2004;71:19-25.

18. Johnson DE, O'Keefe RA, Grandis JR. Targeting the IL-6/JAK/STAT3 signalling axis in cancer. Nat Rev Clin Oncol. 2018 Apr;15(4):234-48.

19. Islam M, Sharma S, Teknos TN.RhoC regulates cancer stem cells in head and neck squamous cell carcinoma by overexpressing IL-6 and phosphorylation of STAT3.PLoS One. 2014 Feb 12;9(2):e88527.

20. Ji CH, Kwon YT. Crosstalk and Interplay between the Ubiquitin-Proteasome System and Autophagy. MOL CELLS. 2017-07-31;40(7):441-9.

21. Zhang L, Chen J, Ning D, Liu Q, Wang C, Zhang Z, et al. FBXO22 promotes the development of hepatocellular carcinoma by regulating the ubiquitination and degradation of p21. J Exp Clin Cancer Res. 2019-02-26;38(1):101.

22. Kitzing TM, Wang Y, Pertz O, Copeland JW, Grosse R. Formin-like 2 drives amoeboid invasive cell motility downstream of RhoC. ONCOGENE. 2010 2010-04-22;29(16):2441-8.

23. Hakem A, Sanchez-Sweatman O, You-Ten A, Duncan G, Wakeham A, Khokha R, et al. RhoC is dispensable for embryogenesis and tumor initiation but essential for metastasis. Genes Dev. 2005;09-01(17):1974-9.. . - ;19(.

24. Thomas P, Pranatharthi A, Ross C, Srivastava S. RhoC: a fascinating journey from a cytoskeletal organizer to a Cancer stem cell therapeutic target. J Exp Clin Cancer Res. 2019;38(1):328. 2019-0724.

25. Hoang MV, Whelan MC, Senger DR. Rho activity critically and selectively regulates endothelial cell organization during angiogenesis. Proc Natl Acad Sci U S A. 2004 Feb;17(7):1874-9. 101(.

26. Aspenstrom P, Fransson A, Saras J. Rho GTPases have diverse effects on the organization of the actin filament system. Biochem J. 2004 Jan 15;377(Pt 2):327-37.

27. Zhang L, Chen J, Ning D, Liu Q, Wang C, Zhang Z, et al. FBXO22 promotes the development of hepatocellular carcinoma by regulating the ubiquitination and degradation of p21. J Exp Clin Cancer Res. 2019-02-26;38(1):101.

28. Kitzing TM, Wang Y, Pertz O, Copeland JW, Grosse R. Formin-like 2 drives amoeboid invasive cell motility downstream of RhoC. ONCOGENE. 2010 2010-04-22;29(16):2441-8.

29. Hakem A, Sanchez-Sweatman O, You-Ten A, Duncan G, Wakeham A, Khokha R, et al. RhoC is dispensable for embryogenesis and tumor initiation but essential for metastasis. Genes Dev. 2005;09-01(17):1974-9.. . -;19(.

30. Thomas P, Pranatharthi A, Ross C, Srivastava S. RhoC: a fascinating journey from a cytoskeletal organizer to a Cancer stem cell therapeutic target. J Exp Clin Cancer Res. 2019;38(1):328. 2019-0724.

31. Hoang MV, Whelan MC, Senger DR. Rho activity critically and selectively regulates endothelial cell organization during angiogenesis. Proc Natl Acad Sci U S A. 2004 Feb;17(7):1874-9. 101(. 
32. Ernst M, Najdovska M, Grail D, Lundgren-May T, Buchert M, Tye H, et al. STAT3 and STAT1 mediate IL-11-dependent and inflammation-associated gastric tumorigenesis in gp130 receptor mutant mice. J Clin Invest. 2008 May;118(5):1727-38.

33. Yu H, Pardoll D, Jove R. STATs in cancer inflammation and immunity: a leading role for STAT3. Nat Rev Cancer. 2009 Nov;9(11):798-809.

34. Yu S, Li G, Wang Z, Wang Z, Chen C, Cai S, et al. The prognostic value of pSTAT3 in gastric cancer: a meta-analysis. J Cancer Res Clin Oncol. 2016-03-01;142(3):649-57.

\section{Table}

Table Characteristics of the study cohort. 


\section{Characteristics}

Case 5 -YSR (\%) $\quad \mathrm{P}$ value $^{\dagger}$ Hazard ratio (95 \% CI) $\quad \mathrm{P}^{\text {value }}{ }^{\ddagger}$

Gender

0.269

$\begin{array}{rrr}\text { Male } & 78 & 36.60 \\ \text { Female } & 35 & 31.97\end{array}$

Age at surgery (years)

0.178

$\begin{array}{lll}<65 & 71 & 37.68 \\ \geq 65 & 42 & 30.93\end{array}$

Tumor location

\begin{tabular}{rcc}
\hline Upper third & 17 & 39.06 \\
\hline Middle third & 15 & 40.73 \\
\hline Lower third & 51 & 38.10 \\
$>2 / 3$ stomach & 30 & 26.217
\end{tabular}

Lauren classification

0.015

$1.275(1.014-1.603)$

0.038

0.683

$\begin{array}{rrr}\text { Intestinal } & 29 & 33.83 \\ \text { Diffuse } & 84 & 35.63\end{array}$

Tumour size $(\mathrm{cm})$

$\begin{array}{lll}<4 & 19 & 50.21 \\ \geq 4 & 94 & 32.13\end{array}$

Type of gastrectomy

0.055

Total $42 \quad 30.60$

$\begin{array}{rll}\text { Proximal subtotal } & 14 & 36.21 \\ \text { Distal subtotal } & 57 & 38.28\end{array}$

Soft tissue invasion

0.093

\begin{tabular}{|c|c|c|c|c|c|c|}
\hline & No & 77 & 32.14 & & & \\
\hline & Yes & 36 & 41.64 & & & \\
\hline \multirow[t]{5}{*}{ pT stage } & & & & 0.964 & & \\
\hline & pT2 & 7 & 34.29 & & & \\
\hline & pT3 & 9 & 35.78 & & & \\
\hline & $\mathrm{pT} 4 \mathrm{a}$ & 90 & 34.99 & & & \\
\hline & pT4b & 7 & 37.57 & & & \\
\hline \multirow[t]{3}{*}{ pN stage } & & & & 0.005 & $1.258(1.092-1.450)$ & 0.002 \\
\hline & pN0 & 23 & 51.52 & & & \\
\hline & pN1 & 14 & 37.93 & & & \\
\hline
\end{tabular}

pT stage

0.299 


\begin{tabular}{rrrrrrr}
\hline pN2 & 25 & 32.40 & & & \\
pN3a & 26 & 28.12 & & & \\
pN3b & 25 & 28.68 & & & \\
\hline RNF180 expression & & & 0.006 & $0.628(0.405-0.974)$ & 0.038 \\
\hline Low & 43 & 29.72 & & & \\
\hline High & 70 & 38.51 & & & \\
\hline
\end{tabular}

Values in parentheses are 95 per cent confidence intervals. *Determined by immunohistochemical staining; †Log rank test; ¥Cox proportional hazards model.

\section{Supplementary Figure Caption}

\section{Supplementary Fig}

mRNA expression levels for genes downstream of STAT3 signaling in GC cells knockdown SATA3 compared with control GC cells.

Figures

A

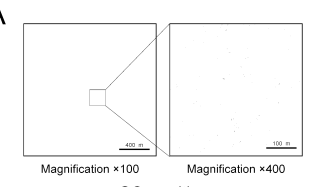

B

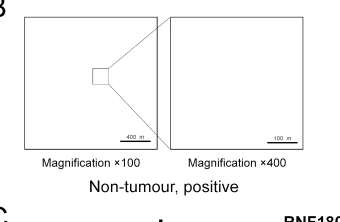

C

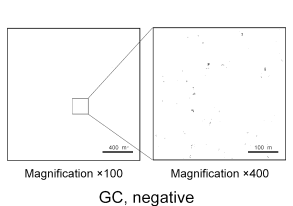

D
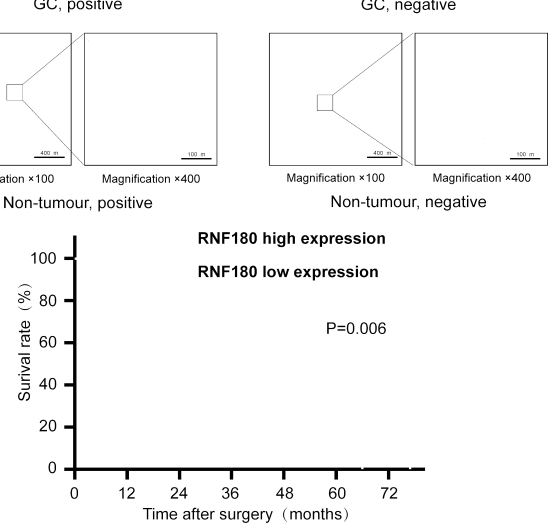

No. at risk

$\begin{array}{lccccccc}\text { RNF180-negative } & 43 & 31 & 22 & 18 & 9 & 4 & 0 \\ \text { RNF180-positive } & 70 & 58 & 50 & 39 & 25 & 19 & 4\end{array}$
E

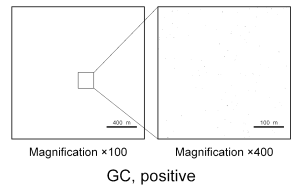

F

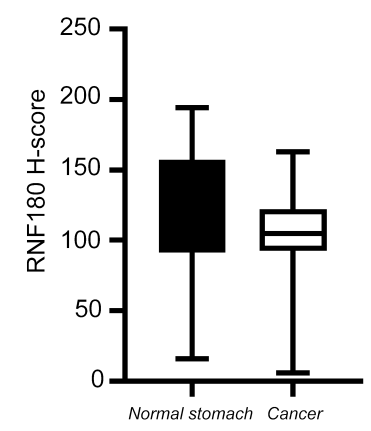

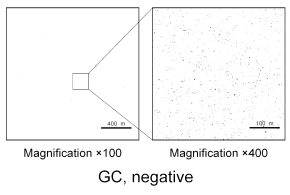

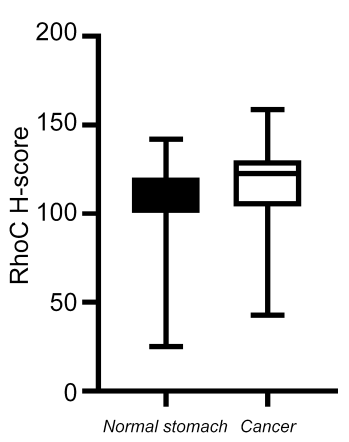

\section{Figure 1}

Immunohistochemical (IHC) staining images of RNF180 and RhoC using human gastric cancer tissue microarrays that contained 113 cancer samples with their matching adjacent histologically normal stomach, original magnification is at $\times 100$ and insets are at $\times 400:(a, b$,$) Examples of RNF180$ 
immunohistochemical staining Showing.(c) Kaplan-Meier survival curves according to expression of RNF180 in GC tissues. $P=0.006$ (log rank test).(d) Ubiquitination label-free quantitative proteomic analysis revealed the ubiquitination level proteins upregulating RNF180 compared with the control group (e) Examples of RhoC immunohistochemical staining showing.(f) Summary graphs of the IHC staining of RNF180 and RhoC results using box-and-whisker blots to depict the smallest value, lower quartile, median, upper quartile, and largest value.

A
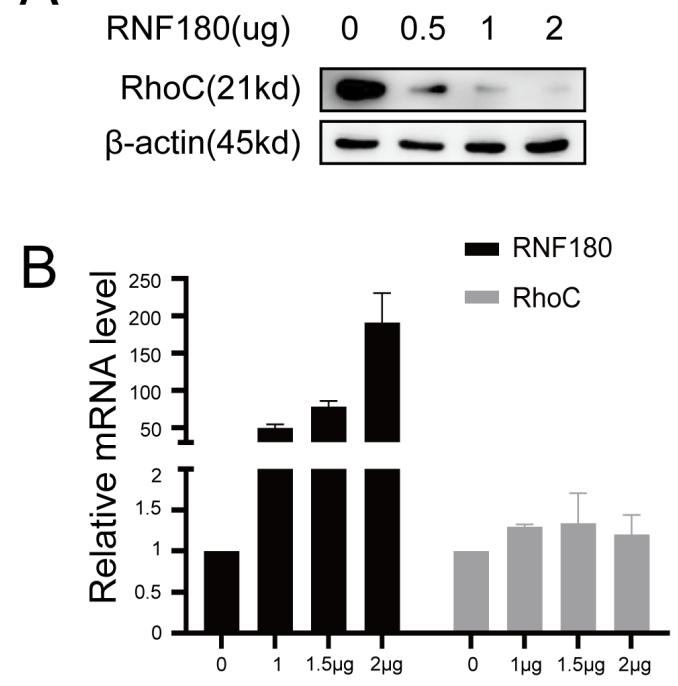

$\mathrm{D}$

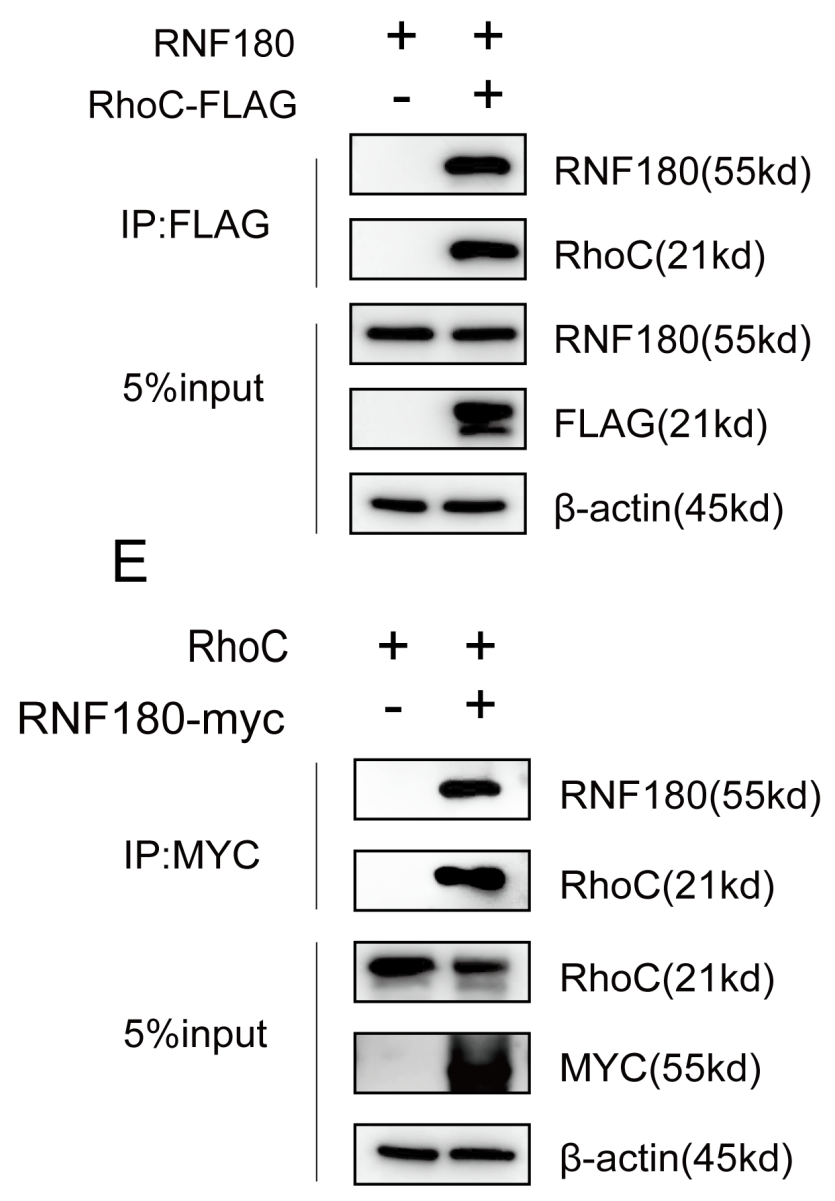

C

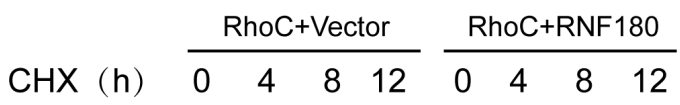

RhoC $(21 \mathrm{kd})$

$\beta$-actin(45kd)

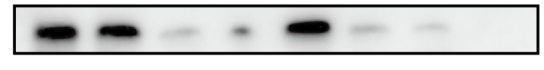

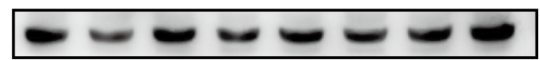

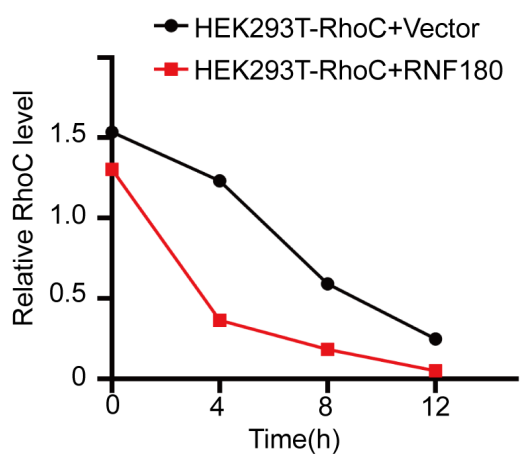

HA-Ub +++

RhoC-FLAG - + +

RNF180 - $\quad$ -

IP:FLAG

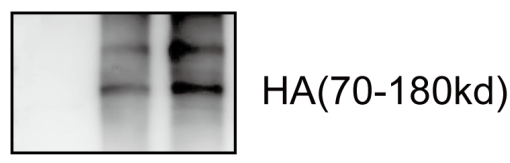

- RhoC(21kd)

- RNF180(55kd)

$\sim \rightarrow \operatorname{RhoC}(21 \mathrm{kd})$

$5 \%$ Input

RNF180(55kd)

$\beta-\operatorname{actin}(45 \mathrm{kd})$

Figure 4 
RNF180 promotes the degradation of RhoC protein by increasing its ubiquitination level and interacts with RhoC.(a)Increasing concentrations of RNF180 plasmid were transfected into HEK293T cells for $36 \mathrm{~h}$ and the protein level RhoC were analyzed by immunoblotting.(b)The mRNA level of RNF180 and RhoC were analyzed by RT-qPCR assay.(c) HEK293T cells were co-transfected by RhoC plasmid together with either RNF180 plasmid or control vector. After $36 \mathrm{~h}$, cells were treated with $50 \mathrm{mg} / \mathrm{ml} \mathrm{CHX}$ at the indicated time point. The cell lysates were subjected to immunoblotting and RhoC expression was quantifified by ImageJ software. (d) The RNF180 plasmid was co-transfected transiently with RhoC-FLAG plasmid or control vector into HEK293T cells and $36 \mathrm{~h}$ later, cells were incubated with $10 \mathrm{mM} \mathrm{MG132}$ for $6 \mathrm{~h}$. Cell lysates were immunoprecipitated to pull down RhoC by the FLAG M2 affinity gel and subjected to immunoblotting. $5 \%$ of cell lysates were used to examine the total expression of RhoC and RNF180. (e) RNF180-FLAG plasmid or control vector was co-transfected transiently with the RhoC plasmid into HEK293T cells and the co-immunoprecipitation assay was performed as described in (d).(f) HA-Ub and RhoC-FLAG plasmids were co-transfected with RNF180 in HEK293T cells. After $36 \mathrm{~h}$, cells were treated with $10 \mathrm{mM} \mathrm{MG} 132$ for $6 \mathrm{~h}$. RhoC-FLAG protein was immunoprecipitated and analyzed by immunoblotting. The poly-ubiquitination level of RhoC was detected by anti-HA antibody. 

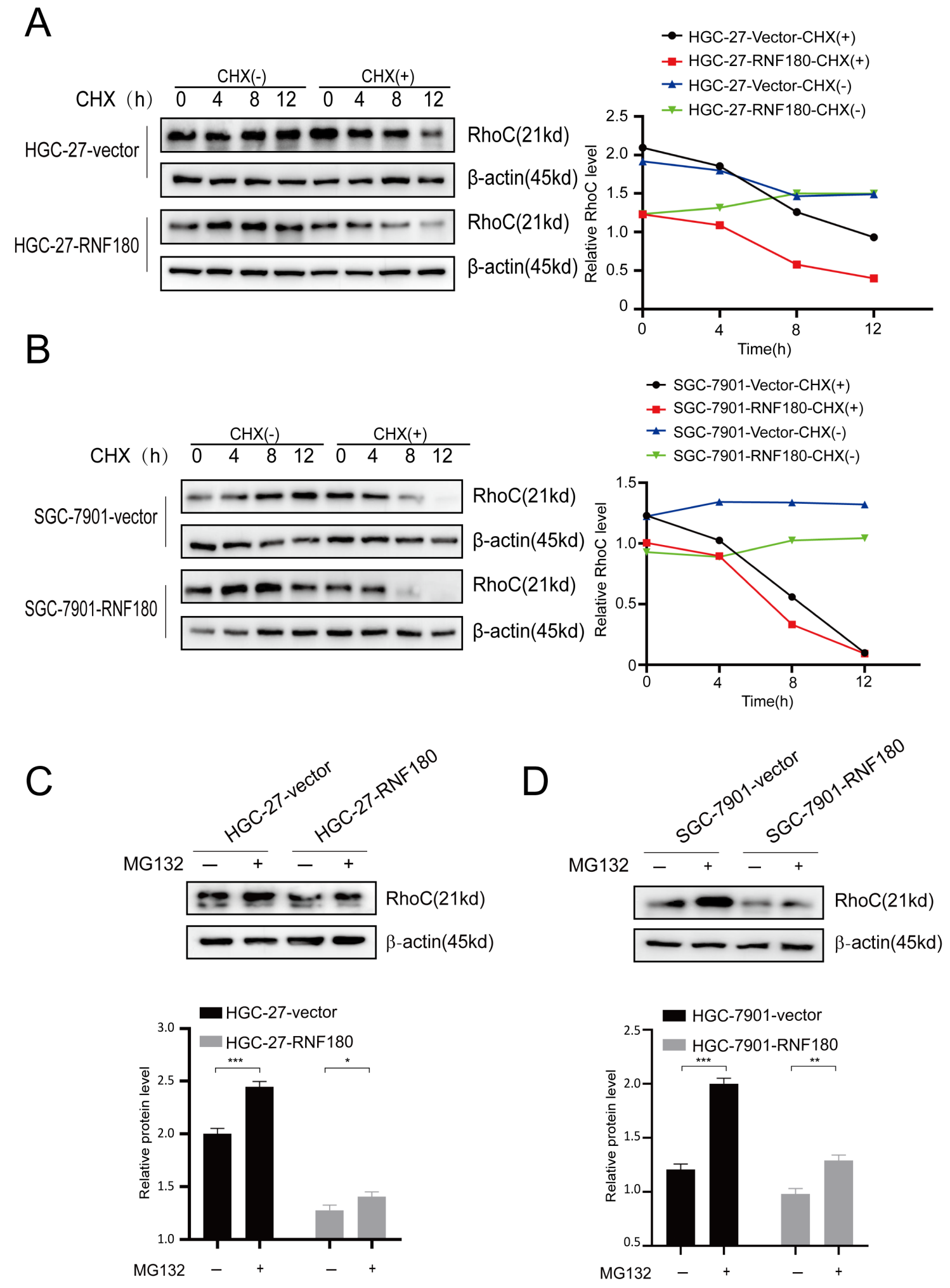

\section{Figure 5}

$(a, b)$ GC cells were transfected by either RNF180 plasmid or control vector. After $36 \mathrm{~h}$, cells were treated with $50 \mathrm{mg} / \mathrm{ml} \mathrm{CHX}$ at the indicated time point. The cell lysates were subjected to immunoblotting and RhoC expression was quantifified bylmageJ software. (c,d)Ubiquitination assay was performed with 10 $\mathrm{mM} \mathrm{MG132}$ for $6 \mathrm{~h}$ in order to further explore the ubiquitination degradation of RhoC and p-STAT3 by RNF180 in GC cells. 
A

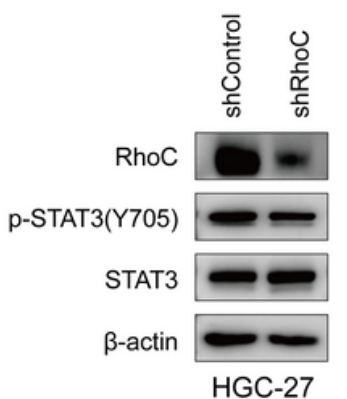

B

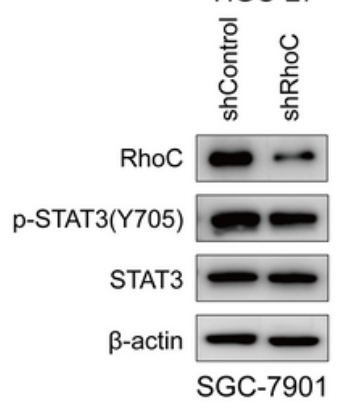

C

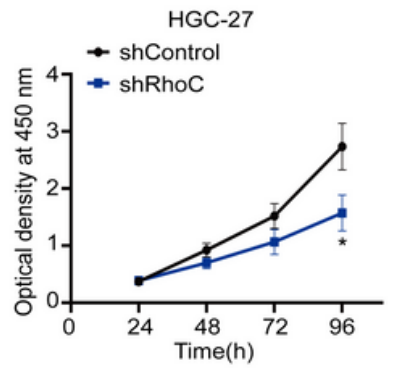

$\mathrm{D}$

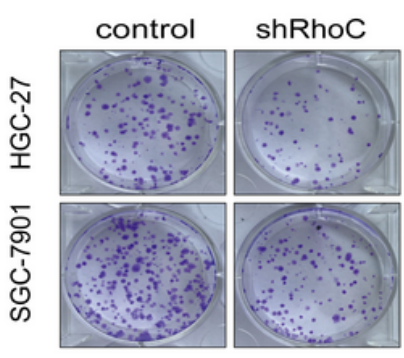

- HGC-27-Control

- HGC-27-shRhoC
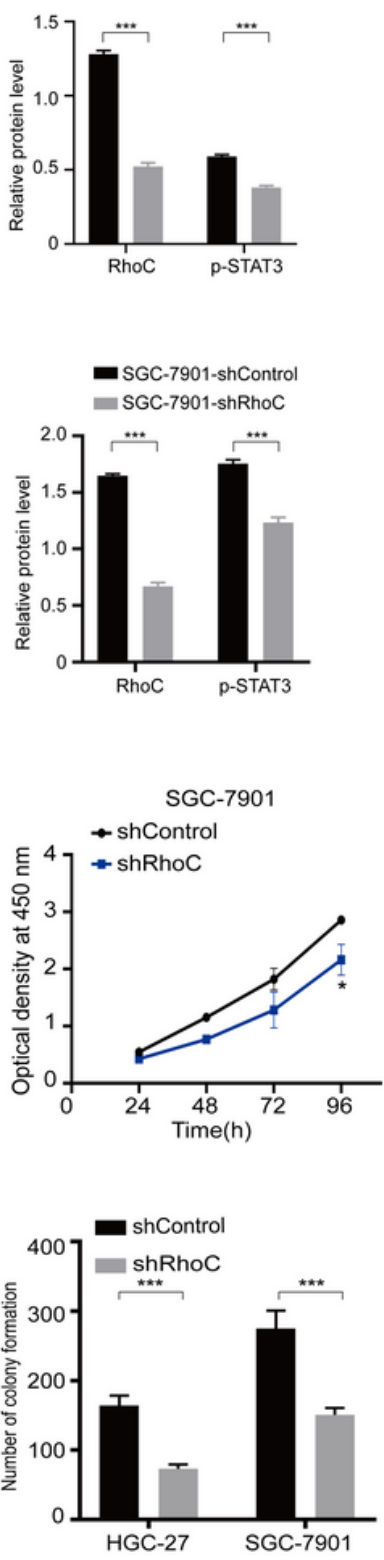

$\mathrm{E}$
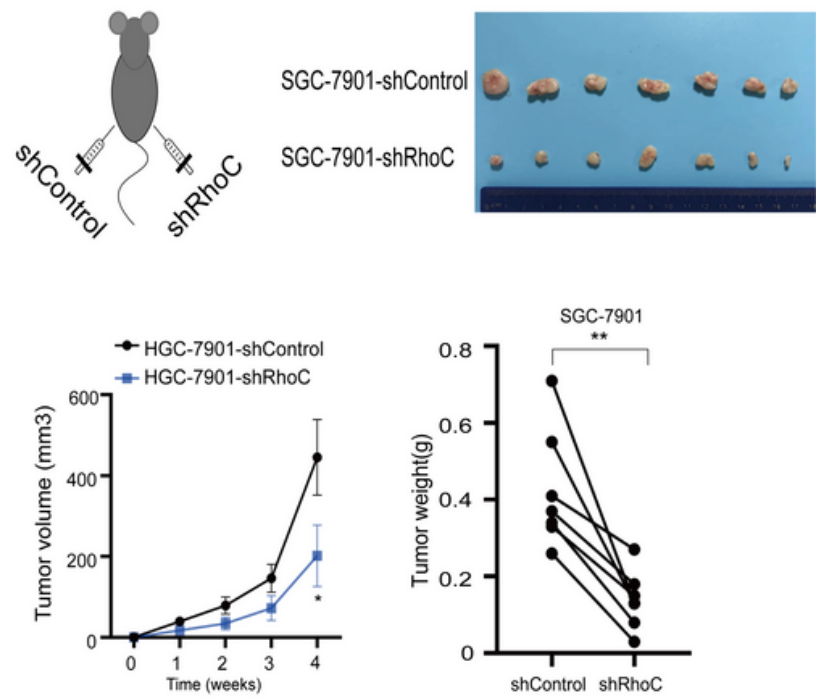

F

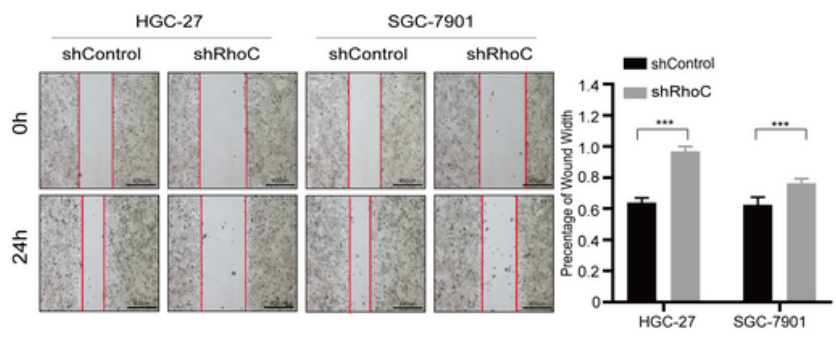

G

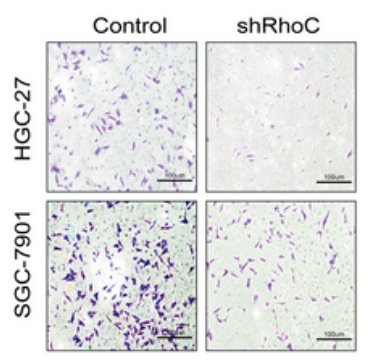

Figure 7

RhoC activity is required for tumor cell proliferation and migration. HGC-27 and SGC-7901 cells were treated with either a control shRNA or an shRNA targeting RhoC.Stably transduced cells were used for analysis of protein expression by western blotting and cell function. $(a, b)$ The protein levels in these established cell lines were verified by western blot.(c)CCK8.(d)colony formation assay.(e)Xenograft tumor formation of SGC-7901 in nude mice.(f)cell-based scratch assay(g)transwell tumour cell invasion assay. 
A
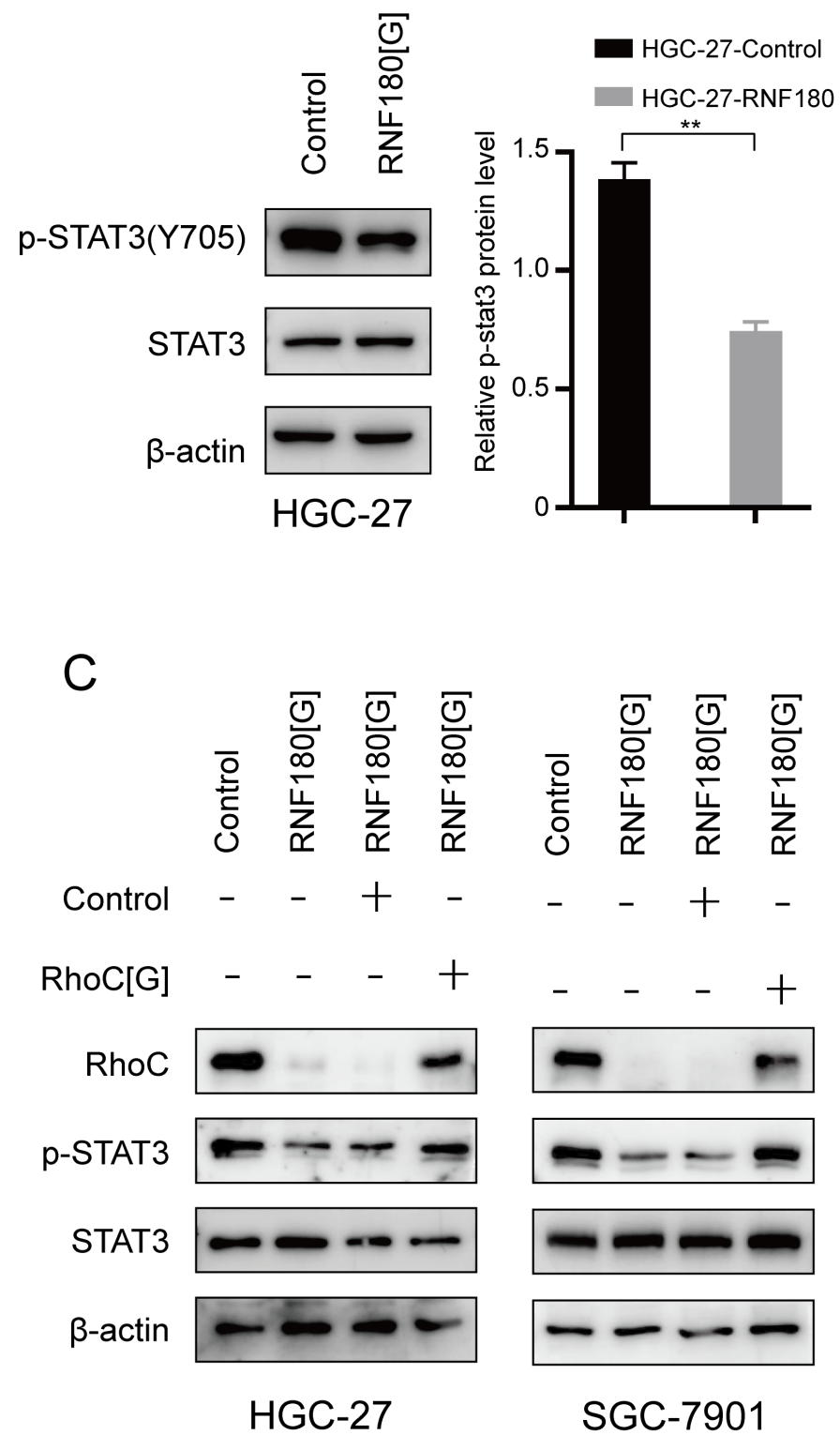
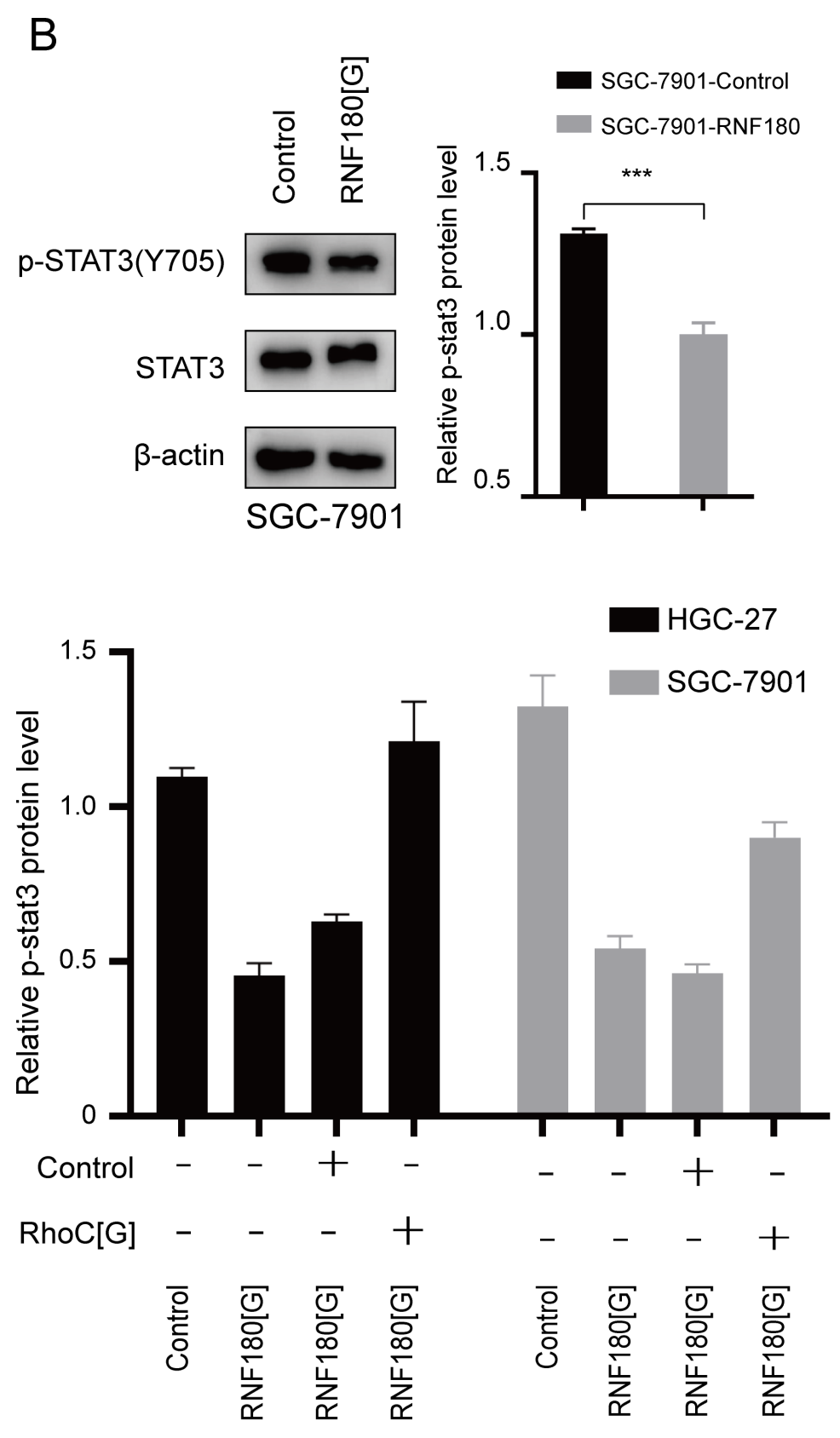

Figure 10

(a,b)HGC-27 and SGC-7901 cells RNF180 compared with control GC cells were established by transiently transfection, respectively.The protein levels in these established cell lines were verified by western blot at $48 \mathrm{~h}$ after transfection.(c)The rescue of RhoC and p-STAT3 that resulted from overexpression of RNF180 by overexpression of RhoC. 


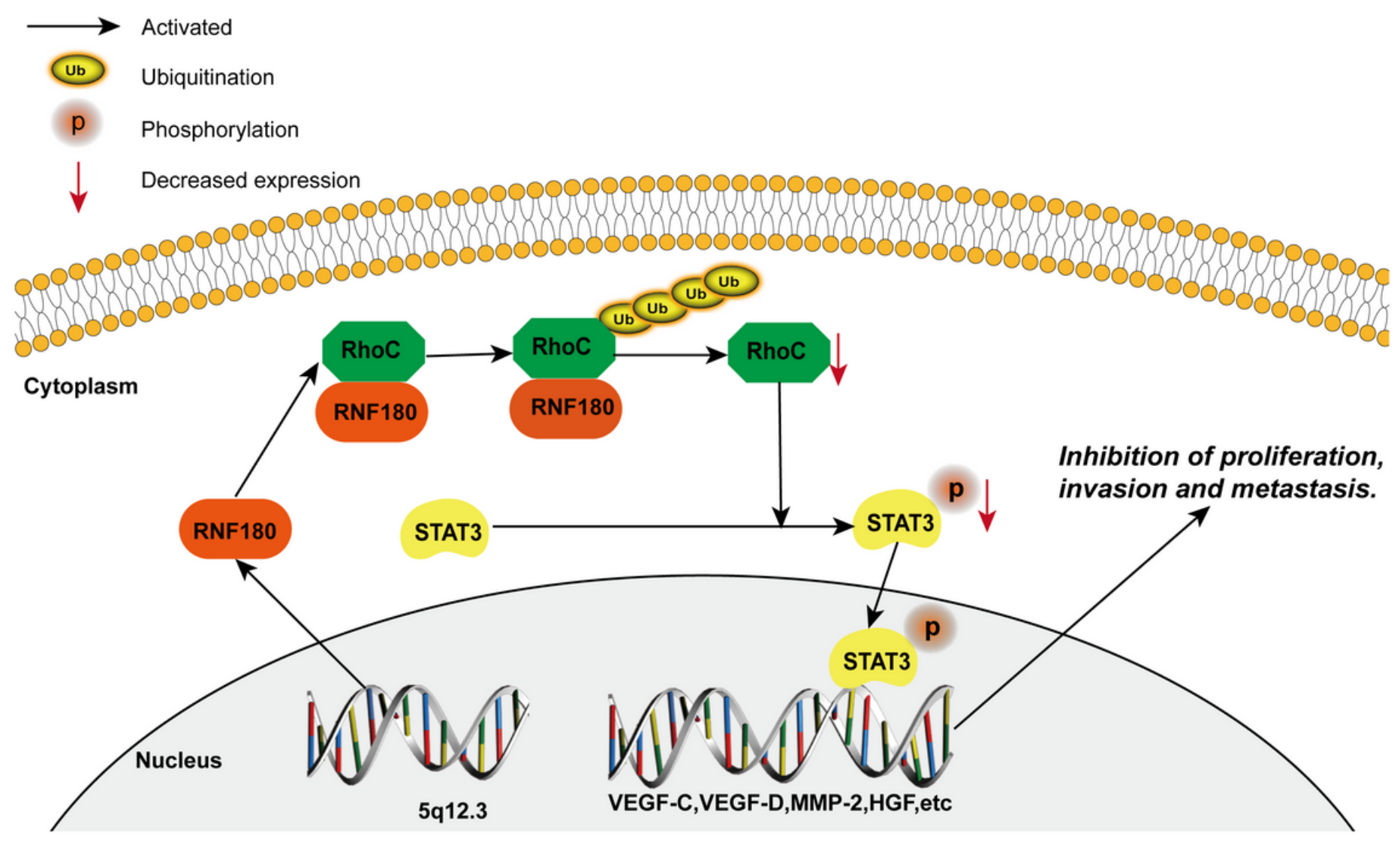

Figure 12

Model depicting role of RNF180 in regulating the expression of protein level of pSTAT3(Y705). Overexpression of RNF180 promotes the degradation of RhoC protein by increasing its ubiquitination level .RhoC could also reduce the protein level of p-STAT3(Y705).Moreover, lowlevel of pSTAT3 could efficiently inhibit the proliferation and invasion of gastric cancer.

\section{Supplementary Files}

This is a list of supplementary files associated with this preprint. Click to download.

- SupplementaryFig.tif

- SupplementaryFig.tif

- SupplementaryTable.doc

- SupplementaryTable.doc 